DOI: 10.2478/romneu-2014-0002

\title{
Assessment of temozolomide action encapsulated in chitosan and polymer nanostructures on glioblastoma cell lines
}

\author{
C. Abrudan ${ }^{1}$, I.S. Florian ${ }^{1}$, A. Baritchii ${ }^{1}$, O. Soritau ${ }^{2}$, S. Dreve ${ }^{3}$, \\ C. Tomuleasa ${ }^{2}$, B. Petrushev ${ }^{2}$ \\ ${ }^{1}$ Department of Neurosurgery, Clinical University Emergency Hospital, Cluj Napoca \\ ${ }^{2}$ Department of Cancer Immunology, Ion Chiricuta Oncology Institute, Cluj Napoca \\ ${ }^{3}$ National Institute for R\&D of Isotopic and Molecular Technologies, Cluj-Napoca
}

\begin{abstract}
Purpose: Glioblastoma multiforme (GBM) remains one of the most devastating diseases known to mankind and affects more than 17,000 patients in the United States alone every year. This malignancy infiltrates the brain early in its course and makes complete neurosurgical resection almost impossible. Recent years have brought significant advances in tumor biology. Many cancers, including gliomas, appear to be supported by cells with stemlike properties. Nanoparticles are excellent candidates to serve as delivery vectors of drugs or biologically active molecules because of their unique chemical and physical properties that result in specific transportation and deposition of such agents in specific organs and tissues..

In the current study we have investigated the in vitro action of nanostructural systems (temozolomide encapsulated in chitosan and polymer nanostructures) on high-grade gliomaderived cancer stem cells (CSCs), with the
\end{abstract}

intention of developing a new therapy to treat specific brain tumors with increased efficacy and minimal toxicity. In vitro cytotoxicity and apoptosis measurements indicated that the drug/vector combination facilitated the ability of the alkylating drug TMZ to alter the resistance of these cancer stem cells, suggesting a new chemotherapy strategy even for patients diagnosed with inoperable or recurrent malignant gliomas

Methods: At the National Institute for $\mathrm{R} \& \mathrm{D}$ of Isotopic and Molecular Technologies form Cluj Napoca were synthesized three types of nanostructures chitosan-TMZ, TMZ-chitosan-PEG (polyethylene glycol), TMZ-chitosan-PPG (polypropylene glycol). Three type of cell lines (Glioma-derived stem, HFL and HUVEC) were treated with the 3 types of nanostructures and the survival rate of the cells was compare to standard therapy (TMZ).

Results: The results showed a reduction in the rate of survival of the tumor cells. Cell proliferation assays clearly demonstrate the differences between 
conventional chemotherapy (TMZ) and temozolomide encapsulated in chitosan and polymer nanostructures.

Conclusion: Nanostructures like chitosan, PEG, PPG are useful as vectors for drugs transport.

Despite combined therapy (surgery, radiotherapy, chemotherapy), currently median patient survival is reduced. The key to improving life expectancy could be an effective therapy targeted, customized for each case. An increasingly important role will be new methods of treatment such as immunotherapy, gene therapy or nanotherapy.

Key words: malignant gliomas, primary tumor cell culture, temozolomide.

\section{Introduction}

Malignant gliomas are responsible for the death of approximately 11,000 patients per year.[1] The standard care for patients diagnosed with high-grade central nervous systems (CNS) gliomas include, postoperatively, temozolomide (TMZ) concomitant and adjuvant to radiotherapy. This therapeutic strategy is, however, associated with high toxicity, limited efficiency and significant side effects. The median length of survival for patients with high-grade primary glial tumors ranges from 11 to 33 months after initial diagnosis and an average of 7 months following recurrence.[2,3]

The failure of current approaches to the treatment of malignant gliomas has been attributed to the existence of a subpopulation of cancer cells malignant glioma cancer stem cells (CSCs), which have the ability to withstand chemotherapeutics and ionizing radiation based on certain of their unique properties: high expression of anti-apoptotic proteins, high expression of $\mathrm{ABC}$ pumps, and remarkable DNA repair capability. [4-6] Traditional therapies, such as DNA alkylating or methylating drugs along with radiation oncology treatments, have low treatment efficacy for these cell types. More complex treatments capable of overcoming the CSCs' ability to eliminate anti-cancer drugs and perform other protective functions are therefore critically needed. For this reason, a combination of traditional treatments and nanotechnologybased approaches offers attractive possibilities. More efficient and less toxic therapeutics that can cross the CSCs protective barriers are urgently needed. In this context, nanomaterials could play an important role based on their unique electronic, optical, magnetic, and structural properties that are found neither in bulk materials nor in single molecules and which are necessary to develop advanced cancer treatments.

\section{Theoretical considerations of nanotherapy}

Nanoparticles are biodegradable or bioresistence polymer matrix, with an average diameter of approx. $200 \mathrm{~nm}$. Nanoparticles are obtained by polymerization of monomers or directly from the processed polymer.

The advantages of nanoparticles are: relatively simple preparation, they ensure protection of the active principles from 
DOI: 10.2478/romneu-2014-0002

chemical and enzymatic degradation (nanocapsules) limiting side effects of active substances and provide transportation and release to the target by the biodegradable matrix. Disadvantages are the high costs of technology (equipment, raw materials) and toxicity of adjuvants [7].

A large area relative to their volume, increased bioavailability, provide controlled release of the active substance and support the intracellular and molecular vectors.

Due to the extremely small size, nanoparticles vectors easily cross biological barriers (blood-brain barrier in our case)[8].

\section{Theoretical considerations of nanotherapy in brain tumors}

Molecular nanodiagnostic. Nanoparticles increased sensitivity and specificity for high-resolution noninvasive medical imaging, at molecular and cellular level (ultrasound, CT, MRI, OI. PET). (PEGchitosan-clorotoxin-fluoroscopic agent nanoparticles may improve the resolution by 10 times)[9].

Nanovaccines are useful for immunotherapy, nanoantibiotherapy offers a lower risk of developing resistance to antibiotics and some nanostructures can be used in neuronal nanoprotection [10].

Transport nanosystems for gene therapy (to replace viruses as vectors) are less likely immunologic and allow the transfer of large quantities of genetic material. A polyglycerol dendrimer polymer has been shown to improve RNA stability and accumulation in brain tumors in animals[7].

Antitumoral nanotherapy PEG dimethacrylate methyl ether methacrylate and iron oxide nanostructures can be used as a biomaterial for the thermal treatment of GBM. Many types of nanoparticles such as biodegradable polymers (PEG, PPG, PBCA) lactic acid loaded with different type of chemotherapy agents increased tumor cytotoxicity and survival in laboratory animals with decreased side effects of cytostatic. Use of nanoparticles can increase the effectiveness of radiotherapy with decrease of side effects [11].

\section{Methods}

At the National Institute for R \& D of Isotopic and Molecular Technologies form Cluj Napoca were synthesized three types of nanostructures chitosan-TMZ, TMZchitosan-PEG (poly-ethylene glycol), TMZchitosan-PPG (polypropylene glycol).

Chitosan [poly(b-(10/4)-2-amino-2deoxy-Dglucose)] is a natural cationic polysaccharide derived from chitin, which is copolymer, a glucosamine and an $\mathrm{N}$ acetyl glucosamine units, combined together [12]. Chitosan is being widely used as a pharmaceutical excipient, comprising a series of polymers varying in their degree of deacylation, molecular weight, viscosity, $\mathrm{pKa}$ etc. The presence of a number of amino groups permits CTS to chemically react with anionic systems, thereby resulting in alteration of 
physicochemical characteristics of reactants and developing new properties of such combinations [1].

Temozolomide (brand names Temodar and Temodal and Temcad) is an oral alkylating agent used for the standard treatment of Grade IV astrocytoma.

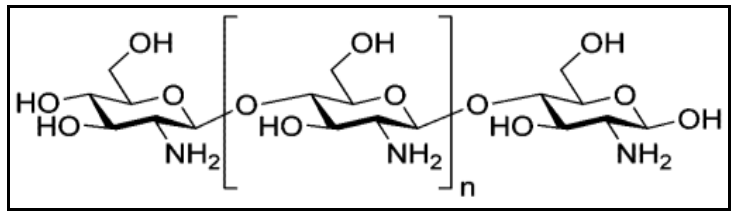

Chitosan structural formula

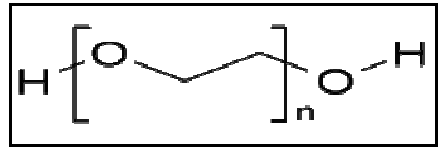

PEG poly-ethylene-glycol

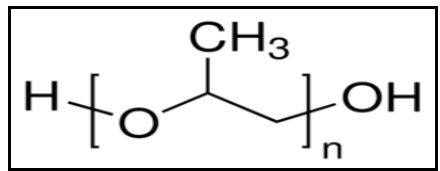

PPG poly-propylene-glycol

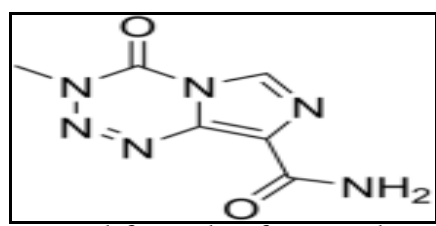

Structural formula of temozolomide

$\mathrm{PEG}+$ chitosan $+\mathrm{Tz}$ (micrometer structures of chitosan with poly-ethylene-glycol and temozolomide.. Spheres of 5-15 microns)
PPG + chitosan $+\mathrm{Tz}$ (micrometer structures of chitosan poly-propylene glycol and temozolomide filaments with a length of 10-50 microns)

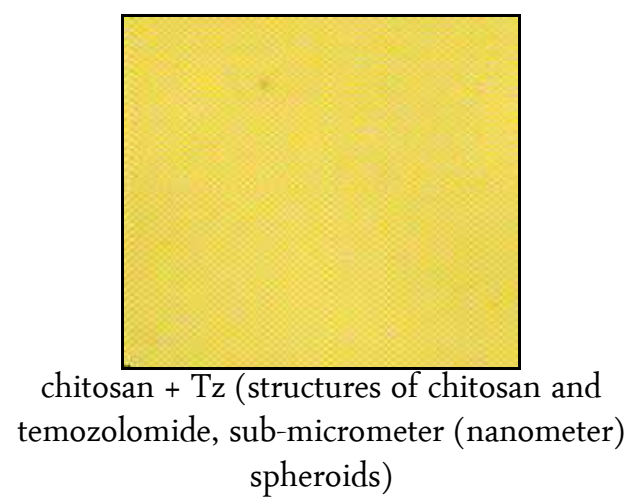

Chitosan (CTS) from crab shells with 85\% deacetylation degree, poly-ethyleneglycol, poly-propylene-glycol, were purchased from Sigma Aldrich.

CTS was dissolved in $1 \%(\mathrm{v} / \mathrm{v})$ acetic acid aqueous solution stirring for 6 hours at $300 \mathrm{C}$ temperature, at final the solution being pale yellow, with a homogenous consistence and aspect. The CTS solution was split in 5 equal volumes, $50 \mathrm{ml}$ each, and to each CTS sample was added $0,5 \mathrm{ml}$ linking additive agent and $10 \mathrm{mg}$ powder from Temodal. Calculations assured that each sample contains $10 \mathrm{mg}$ TMZ.

We obtain a temozolomide concentration of $50 \mathrm{micromol} / \mathrm{l}$.

The solutions were further stirred for 30 min and then ultrasonically treated in an 
Elmasonic E60H ultrasonic bath for 360 $\min$.

Microspheres of CTS-based polyelectrolyte complex containing TMZ/linker were characterized by FTIR electronic microscope connected to FTIR JASCO 6100 spectrometer.

The malignant glioma cancer stem cells (CSCs), used in this study were isolated from a glioblastoma multiforme biopsy. Briefly, after mechanical dissociation of tumor tissue, the fragments were placed in $1 \mathrm{ml}$ of fetal calf serum (FCS). After three hours, $3 \mathrm{ml}$ of DMEM/F-12 medium supplemented with $15 \%$ FCS was added to the dish. After reaching a subconfluent monolayer, cells were detached using trypsin/ EDTA and resuspended in a serum-free media: DMEM/F12 (1:1) medium, supplemented with $15 \mathrm{ng} / \mathrm{ml}$ basic fibroblast growth factor (bFGF), 20 $\mathrm{ng} / \mathrm{ml}$ epidermal growth factor (EGF), $2 \mathrm{mM} / 1$ L-glutamine, $4 \mathrm{U} / 1$ insulin growth factor-1 (IGF-1) and B 27 supplement (1:50) (Sigma Aldrich). Isolated and expanded cells revealed some stem-cell specific features, such as the expression of cellular markers (CD133, CD105, CD90, Nanog, Oct 3/4 (immunocytochemistry) expression of specific genes, such as : CXCR4, nestin, glial fibrillary acidic protein (GFAP), and neurofilament protein (NF) (reverse transcription-PCR). Cells also displayed a high proliferative potential despite chemotherapy and irradiation and also had the ability to form spheroids in suspension.
The three type of cell lines (Gliomaderived stem, HFL-Normal fibroblasts human isolated from lung and endothelial cell line HUVEC-Human Umbilical Vein endothelial cell.) were treated with the 3 types of nanostructures and the survival rate of the cells was compare to standard therapy (TMZ).

We used three cell lines to study the difference between tumor cells and normal cell lines (fibroblasts and endothelial cells).

The four compounds (PEG + chitosan + $\mathrm{Tz}, \mathrm{Tz}+\mathrm{PPG}+$ chitosan, chitosan $+\mathrm{Tz}$ and TZ) after binding were filtered through a $220 \mathrm{~nm}$ filter sterilized

Stem cells are in the exponential phase of cell growth they are detached by exposure for $5 \mathrm{~min}$ in $0.25 \%$ trypsin EDTA after 3 washes with PBS. Trypsin is inactivated by the addition of culture medium with $10 \%$ fetal calf serum, and the cell suspension centrifuged $5 \mathrm{~min}$ at 1100 rpm. Cell viability is checked by trypan blue $0.4 \%$. The cells are counted with a Thoma chamber.

The 3 cell lines (GM 1, HFL and HUVEC) after being counted are seeded in 96-well plates each 7500 cells / well and suspended in $200 \mathrm{ml}$ medium; after $24 \mathrm{~h}$ cells were subjected to treatment that joined us and after a further 24 hours MTT assay was performed

MTT test. Twenty-four hours after therapy culture medium was aspirated and the cells will be exposed to $100 \mathrm{ml}$ solution of MTT $1 \mathrm{mg} / \mathrm{ml}$ (tetrazolium Bromide Thyazolyl Blue) for 1 hour at $37^{\circ} \mathrm{C}$. MTT is a tetrazolium salt which is converted in 
cellular mitochondria of viable cells into a formazan compound dark blue colored, insoluble in aqueous solutions. After the incubation period, MTT solution is aspirated from the wells and formazan crystals were dissolved with DMSO $150 \mu \mathrm{l} /$ well (dimethyl sulfoxide) obtaining a color reaction. For measuring the optic density, boards are analyzed at $492 \mathrm{~nm}$ using a plate reader BioTek Synergy 2 . Each determination shall be made in triplicate.

\section{Statistical analysis}

Statistical significance values were obtained using a one-way analysis of variance (ANOVA), with 95\% confidence (C.I.) level, using GraphPad Prism 5 statistics program (GraphPad Inc, San Diego, CA, USA). Bonferroni's multiple comparison test was considered statistically significant at $\mathrm{p}<0.05$. All experiments were performed in triplicate.

\section{Results}

In our experiments,. there was an impotant difference between TMZ alone (at the same concentration) and the control sample on the one hand and the three types of nanostructures studied (PEG + chitosan + Tz, Tz + PPG + chitosan, chitosan $+\mathrm{Tz}$ ) on the other hand for each cell type (Glioma-derived stem, HFL and HUVEC). Figure 1

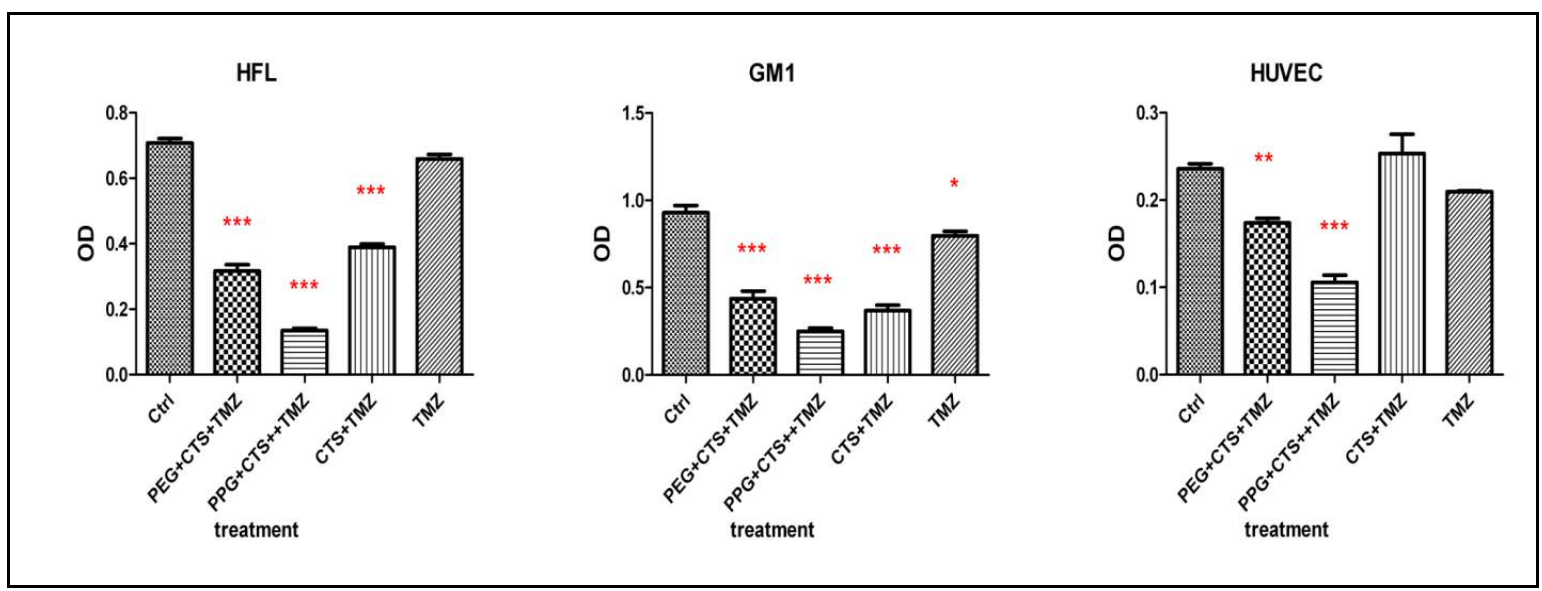

Figure 1

Survival chemotherapy graphics for the three type of cell lines (Glioma-derived stem, HFL-Normal fibroblasts human isolated from lung and endothelial cell line HUVEC-Human Umbilical Vein endothelial cell.) treated with the 3 types of nanostructures and standard therapy (TMZ). The vertical axis represents the optical density (remaining cell population) and the horizontal axis the various therapy options.

HFL

\begin{tabular}{|l|rrrrr|}
\hline & Mean & \multicolumn{4}{|c|}{ Significant? } \\
Bonferroni's Multiple Comparison Test & Diff. & $\mathrm{t}$ & $\mathrm{P}<0.05$ ? & Summary & 95\% CI of diff \\
\hline Ctrl vs PEG+CTS+TMZ & 0.3903 & 20.68 & Yes & $* * *$ & 0.3282 to 0.4523 \\
Ctrl vs PPG+CTS+TMZ & 0.5728 & 30.35 & Yes & $* * *$ & 0.5107 to 0.6348 \\
\hline
\end{tabular}


DOI: 10.2478/romneu-2014-0002

\begin{tabular}{|l|rrcrr|}
\hline Ctrl vs CTS+TMZ & 0.3183 & 16.86 & Yes & $* * *$ & 0.2562 to 0.3803 \\
Ctrl vs TMZ & 0.04825 & 2.557 & No & ns & -0.01376 to 0.1103 \\
PEG+CTS+TMZ vs PPG+CTS+TMZ & 0.1825 & 9.671 & Yes & $* * *$ & 0.1205 to 0.2445 \\
PEG+CTS+TMZ vs CTS+TMZ & -0.07200 & 3.815 & Yes & $*$ & -0.1340 to -0.009987 \\
PEG+CTS+TMZ vs TMZ & -0.3420 & 18.12 & Yes & $* * *$ & -0.4040 to -0.2800 \\
PPG+CTS+TMZ vs CTS+TMZ & -0.2545 & 13.49 & Yes & $* * *$ & -0.3165 to -0.1925 \\
PPG+CTS+TMZ vs TMZ & -0.5245 & 27.79 & Yes & $* * *$ & -0.5865 to -0.4625 \\
CTS+TMZ vs TMZ & -0.2700 & 14.31 & Yes & $* * *$ & -0.3320 to -0.2080 \\
\hline
\end{tabular}

GM1

\begin{tabular}{|c|c|c|c|c|c|}
\hline \multirow[b]{2}{*}{ Bonferroni's Multiple Comparison Test } & \multirow{2}{*}{$\begin{array}{r}\text { Mean } \\
\text { Diff. }\end{array}$} & \multicolumn{2}{|c|}{ Significant? } & \multirow{2}{*}{\multicolumn{2}{|c|}{$95 \%$ CI of diff }} \\
\hline & & $\mathrm{t}$ & $\mathrm{P}<0.05 ?$ & & \\
\hline Ctrl vs PEG+CTS+TMZ & 0.4933 & 10.53 & Yes & $* * *$ & 0.3394 to 0.6471 \\
\hline Ctrl vs PPG+CTS+TMZ & 0.6788 & 14.49 & Yes & $* * *$ & 0.5249 to 0.8326 \\
\hline Ctrl vs CTS+TMZ & 0.5603 & 11.96 & Yes & *** & 0.4064 to 0.7141 \\
\hline Ctrl vs TMZ & 0.1325 & 2.829 & No & & -0.02138 to 0.2864 \\
\hline $\mathrm{PEG}+\mathrm{CTS}+\mathrm{TMZ}$ vs PPG+CTS+TMZ & 0.1855 & 3.961 & Yes & & $\begin{array}{r}0.03162 \text { to } 0.3394 \\
-0.08688 \text { to }\end{array}$ \\
\hline PEG+CTS+TMZ vs CTS+TMZ & 0.0670 & 1.431 & No & ns & 0.2209 \\
\hline PEG+CTS+TMZ vs TMZ & -0.3608 & 7.704 & Yes & & -0.5146 to -0.2069 \\
\hline PPG+CTS+TMZ vs CTS+TMZ & -0.1185 & 2.531 & No & & -0.2724 to 0.03538 \\
\hline $\mathrm{PPG}+\mathrm{CTS}+\mathrm{TMZ}$ vs TMZ & -0.5463 & 11.66 & Yes & $* * *$ & -0.7001 to -0.3924 \\
\hline CTS+TMZ vs TMZ & -0.4278 & 9.134 & Yes & *** & -0.5816 to -0.2739 \\
\hline
\end{tabular}

HUVEC

\begin{tabular}{|l|rrrrr|}
\hline & \multicolumn{5}{|c|}{ Signific } \\
Bonferroni's Multiple Comparison Test & ant P Summar \\
\cline { 2 - 7 } Ctrl vs PEG+CTS+TMZ & Mean Diff. & $\mathrm{t}<0.05$ ? & y & $95 \%$ CI of diff \\
Ctrl vs PPG+CTS+TMZ & 0.1303 & 8.232 & Yes & $* * *$ & 0.01001 to 0.1140 \\
Ctrl vs CTS+TMZ & -0.01775 & 1.122 & No & ns & -0.06974 to 0.03424 \\
Ctrl vs TMZ & 0.02625 & 1.659 & No & ns & -0.02574 to 0.07824 \\
PEG+CTS+TMZ vs PPG+CTS+TMZ & 0.06825 & 4.313 & Yes & $* *$ & 0.01626 to 0.1202 \\
PEG+CTS+TMZ vs CTS+TMZ & -0.07975 & 5.040 & Yes & $* *$ & -0.1317 to -0.02776 \\
PEG+CTS+TMZ vs TMZ & -0.03575 & 2.259 & No & ns & -0.08774 to 0.01624 \\
PPG+CTS+TMZ vs CTS+TMZ & -0.1480 & 9.354 & Yes & $* * *$ & -0.2000 to -0.09601 \\
PPG+CTS+TMZ vs TMZ & -0.1040 & 6.573 & Yes & $* * *$ & -0.1560 to -0.05201 \\
CTS+TMZ vs TMZ & 0.04400 & 2.781 & No & ns & -0.007993 to o.09599 \\
\hline
\end{tabular}



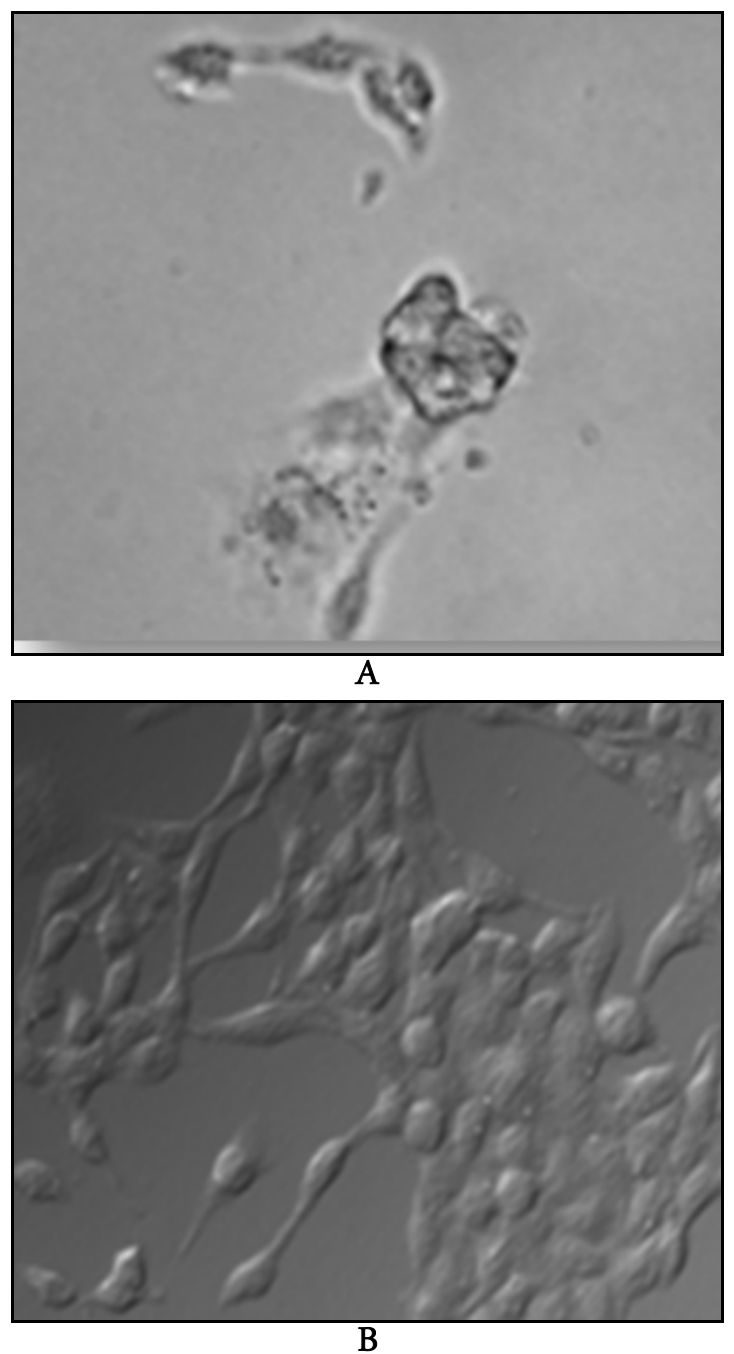

Figure $2(\mathrm{~A})$

A typical pre-apoptotic cell after $24 \mathrm{~h}$ incubation with chitosan+TMZ (PlasDIC phase contrast, magnification 400x), and (B) in comparison with control (cells culture without chitosan+TMZ), (white light microscopy, PlasDIC contrast phase, magnification 400x)

After cell GM1 treatement with chitosan+TZM autophagic cells were observed.

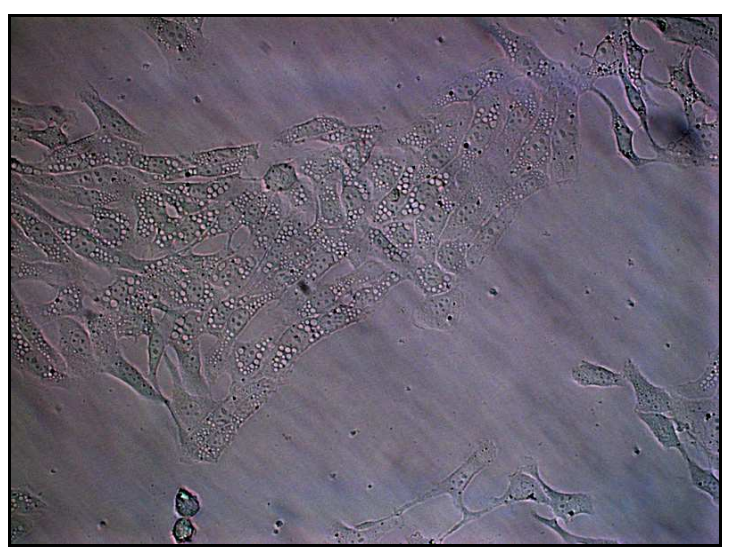

Figure 3

Autophagic cell GM1with intracytoplasmic vacuoles it was observed for chitosan+TZM nanoparticles.

(phase contrast, magnification $\mathrm{x} 400$ )

\section{Discussion and conclusion}

Long-term survival of patients diagnosed with high-grade gliomas remains poor, with population-based studies estimating that the 3-year survival rate is under 5\%. [22-25]. Conventional treatment for newly diagnosed malignant gliomas was traditionally consisted of initial surgical resection followed by fractionated external beam RT, with or without chemotherapy usually using regimens containing alkylating agents. Until recently the benefit of chemotherapy in this setting remained controversial but TMZ, an oral alkylating agent, has proven to be efficient, primarily in the recurrent setting.

The failure of current approaches to the treatment of malignant gliomas has been attributed to the existence of a subpopulation of cancer cells malignant glioma cancer stem cells (CSCs), which have the ability to withstand chemotherapeutics and ionizing radiation 
DOI: 10.2478/romneu-2014-0002

based on certain of their unique properties: high expression of anti-apoptotic proteins, high expression of $\mathrm{ABC}$ pumps, and remarkable DNA repair capability.[13-15] Traditional therapies, such as DNA alkylating or methylating drugs along with radiation oncology treatments, have low treatment efficacy for these cell types. More complex treatments capable of overcoming the CSCs ability to eliminate anti-cancer drugs and perform other protective functions are therefore critically needed. For this reason, a combination of traditional treatments and nanotechnologybased approaches offers attractive possibilities. More efficient and less toxic therapeutics that can cross the CSCs protective barriers are urgently needed. In this context, nanomaterials could play an important role based on their unique electronic, optical, magnetic, and structural properties that are found neither in bulk materials nor in single molecules and which are necessary to develop advanced cancer treatments.

Similarities between the self-renewal mechanisms of stem cells and cancer cells have led to the new concept of cancer stem cells (CSCs). Over the course of the past 10-15 years, there has been increasing evidence to support the cancer stem cell hypothesis, which postulates that CSCs are responsible for tumor initiation, metastasis, and resistance to treatment. It is now generally believed that a tumor has its origin in CSCs, which come either from transformed tissue stem cells or from transformed progenitor cells that have regained self-renewal activity.[16] These rare CSCs could be crucial in controlling and curing cancer: through asymmetric division, CSCs drive tumor growth and evade therapy with the help of traits shared with normal stem cells such as quiescence, self-renewal ability, and multidrug resistance pump activity.[17] These cells were first identified in hematologic cancers, but recently have been isolated from solid tumors. CSCs are tumor initiating cells in immunocompromised mice and have the ability to generate heterogeneous cancer cell populations.[18]

The gold standard assay to determine whether a stem cell is or not a CSC involves serial transplantation in animal models. Potential surface markers of CSCs include the following: CD133, aldehyde dehydrogenase 1 (ALDH1), CD44, and CD24. Efflux of Hoechst or Rhodamine dyes, also referred to as Side Population (SP), have also been used to identify putative CSCs. However, these markers have certain limitations in that they fail to identify all CSCs and merely designate a subpopulation that is enriched for clonogenic and tumorigenic activity. Also, not all cells with a CSC marker phenotype behave as CSCs. Most markers for separating CSCs were chosen due to their expression on normal stem cells of certain tissues, and, interestingly, there are a number of molecules that are commonly expressed in normal and cancer stem cells that lead to different phenomena depending on the local environment.[19] 
Using Bonferroni's Multiple Comparison Test, we found statistically significant results $(\mathrm{P}<0.05)$ between the CSCs (control) and the CTR+ $\mathrm{PEG}+\mathrm{CTS}+\mathrm{TMZ}$ (95? CI of 0,3394 to 0,6471), CTR+PPG-CTS-TMZ (95\% Cl of $0,5249$ to 0,83260$)$, CTR+CTS-TMZ (95\% of 0,4064 to 0,7141$)$. When one or more initiating genetic changes appeared at the progenitor level, all of the downstream cells continued this change. In one particular case, it is possible that a daughter cell acquired not only the properties of the stem cell, but also the additional alterations that allow the glioma to progress to the next step and invade surrounding tissues. Due to the small population of glioblastoma-derived stem cells, the malignant gliomas have a negative response to various conventional treatments.[20] The killing efficiency of the PEG+CTS+TMZ, PPG-CTS-TMZ, CTS-TMZ nanostructures on glioblastomaderived stem cells is better compared with the drug temozolomide, alone.

Our proposed nanoscaled drug delivery system offers also a new chemotherapy strategy for patients diagnosed with unresectable or recurrent malignant gliomas. Current therapies are not yet curative, as CSCs may survive as a result of the increased efflux of chemotherapy agents due to ABCG2 cell membrane proteins and increased DNA repair.[21]

Cancer cells are very complex biological structures that perform functions ranging from invasion or metastasis to the elimination of anticancer drugs from the cell membrane. Although the exact mechanisms need to be explored further, combining advances in fundamental oncology and nanotechnology offers the opportunity to significantly impact future diagnostics and therapeutics. We have shown that drug delivery vectors based on chitosan and polymers have the ability to deliver temozolomide (TMZ), a cytostatic drug, to treat malignant gliomas. Our studies have shown that a novel drug delivery has low toxicity and the ability to internalize TMZ.

Malignant gliomas are highly infiltrative and lethal cancers of the central nervous system. The highly infiltrative nature of glioma cells often renders a complete surgical removal impossible and inevitably will lead to tumor recurrence.

The attention of the scientific community is currently focused on nanoparticles, a novel vector for the delivery of anticancer drugs to target cancer cells. These particles have many advantages which recommend it over classically administered drugs. Due to their submicroscopic size and modifiability, nanoparticles have an enhanced access to cancer cells, being able to maintain high concentrations of drugs in target tissues. Because of their special distribution, drugloaded nanoparticles may even have a decreased risk of systemic adverse effects which normally occur at increased drug doses, while locally maintaining effective concentrations. Nanoparticles loaded with various cytostatics may prove to have numerous additional advantages. The 
increased drug concentrations may also be explained by the facilitated penetration of the particles in the tumor through endocytosis, which has the advantage of bypassing the transporter- mediated drug internalization systems. Moreover, the intracellular drug concentration increases in spite of tumor cell multidrug resistance protein activity, which may result in better tumor-level effect of the drug in spite of multidrug resistant tumor phenotype. Another positive viewpoint of using nanoparticles is their capacity to cross the blood brain barrier, which shapes new directions for drug delivery into the brain. Most drugs partially cross the blood brain barrier, but its incorporation into nanoparticles may enhance its passage and increase the relative amount of drug reaching brain tissue. Therefore, the use of loaded nanoparticles could be of paramount importance for glioblastomas, aggressive tumors with a very dismal prognosis, for which temozolomide has been entitled "the most clinically relevant drug ever reported for targeting of gliomainitiating cells".[25]

Nanostructures like chitosan, PEG, PPG are useful as vectors for temozolomide transport.

Our study shows a net decrease of cell population by treating them with drugnanostructures. Cell population decrease was more important in tumor cell cultures (GM1) compared with normal cells fibroblasts (HFL) or endothelial cells (HUVEC). The best response was obtained for chitosan-Tz, considering that drug chemotherapy should be aggressive on tumor cells and less aggressive on normal cells.

Our study tries to find new drugs for the treatment of glioblastoma. Results must be confirmed by in vivo studies.

Despite combined therapy (surgery, radiotherapy, chemotherapy), currently median patient survival is $10-12$ months. The key to improving life expectancy could be an effective therapy targeted, customized for each case. An increasingly important role will be new methods of treatment such as immunotherapy, gene therapy or nanotherapy.

\section{References}

1. Behin A, Hoang-Xuan K, Carpentier AF, Delattre JY. Primary brain tumors in adults. Lancet 2003; 361: 323331.

2. Wen PY, Kesari S. Malignant gliomas in adults. N Engl J Med 2008; 359: 492-507.

3. Stupp R, Hottinger AF, van den Bent $M J$ et al. Frequently asked questions in the medical management of high-grade gliomas: a short guide with practical answers. ESMO educational book. Ann Oncol 2008; 19 (Suppl 7): vii209-vii216.

4. Stupp R, Tonn JC, Brada M, Pentheroudakis G. High-grade malignant gliomas: ESMOclinical practice guidelines for diagnosis, treatment and follow-up. Ann Oncol 2010; 21 (Suppl 5): v190-v193.

5. Stupp R, Mason WP, van den Bent MJ et al. Radiotherapy plus concomitant adjuvant temozolomide for glioblastoma. N Engl J Med 2005; 352: 987-996.

6. Tomuleasa C, Soritau O, Rus-Ciuca D et al. Functional and molecular characterization of glioblastoma multiforme-derived cancer stem cells. J BUON 2010; 15: 583-591.

7. Jain KK: Potential of nanobiotechnology in management of glioblastoma multiforme. In: Glioblastoma: Molecular Mechanisms of Pathogenesis and Current Therapeutic Strategies. Ray SK (Ed.). Springer, NY, USA, 399-419 (2010).

8. Jain KK: Potential of nanobiotechnology in 
management of glioblastoma multiforme. In: Glioblastoma: Molecular Mechanisms of Pathogenesis and Current Therapeutic Strategies. Ray SK (Ed.). Springer, NY, USA, 399-419 (2010).

9. Wang J, Yong WH, Sun Y et al.: Receptor-targeted quantum dots: fluorescent probes for brain tumor diagnosis. J. Biomed. Opt. 12, 044021 (2007).

10. Jain KK: Textbook of Personalized Medicine. Springer, NY, USA (2009).

11. Maier-Hauff K, Ulrich F, Nestler D et al.: Efficacy and safety of intratumoral thermotherapy using magnetic iron-oxide nanoparticles combined with external beam radiotherapy on patients

12. Svirshchevskaya E.V. et al. - European J. of Medicinal Chemistry 445 (2009) 2030-2037.

13. Eyler CE, Rich JN. Survival of the fittest: cancer stem cells in therapeutic resistance and angiogenesis. J. Clin. Oncol. 26(issue number??), 2839-2845 (2008).

14. Li X, Lewis MT, Huang J et al. Intrinsic resistance of tumorigenic breast cancer cells to chemotherapy. J. Natl. Cancer Inst. 100 (issue?), 672-67 (2008).

15. Diehn M, Cho RW, Lobo NA et al. Association of reactive oxygen species levels and radioresistance in cancer stem cells. Nature 458 (issue?), 780-783 (2009).

16. Dittmar T, Nagler C, Schwitalla S, Reith G, Niggemann B, Zänker KS. Recurrence cancer stem cells-made by cell fusion? Med Hypotheses 73(4), 542-7 (2009).
17. Fabian A, Barok M, Vereb G, Szollosi J. Die Hard: Are Cancer Stem Cells the Bruce Willises of Tumor Biology? Cytometry; Part A 75A, 67-74 (2009).

18.Keysar S B, Jimeno A. More than Markers: Biological Significance of Cancer Stem Cell-Defining Molecules. Mol Cancer Ther 9 (issue number?):2450-2457 (2010).

19. Iwasaki H, Suda T. Cancer stem cells and their niche. Cancer Sci. 100(7), 1166-72 (2009)

20. Florian IS, Tomuleasa C, Soritau O et al. Cancer stem cells and malignant gliomas. From pathophysiology to targeted molecular therapy. J Buon. 16(1), 16-23 (2011).

21. Frosina G. DNA repair and resistance of gliomas to chemotherapy and radiotherapy. Mol Cancer Res 7(7), 989-999 (2009).

22. Nino Lomadze, Hans Jorg Schneider - Tetrahedron 6136 (2005) 8694-8698.

23. O. Soritau, C. Tomuleasa, M. Aldea et al. Metformin plus temozolomide-based chemotherapy as adjuvant treatment for WHO grade III and IV malignant gliomas Journal of BUON 16 (2011): 282-289,

24. Gerster ER, Batchelor TT. Imaging and response criteria in gliomas.Curr Opin Oncol 2010; 22: 598-603. 25. Grossman SA, Ye X, Piantadosi S et al. Survival of patients with newly diagnosed glioblastoma treated with radiation and temozolomide in research studies in the United States. Clin Cancer Res 2010; 16: 2443-2449. 